\title{
Caracterização do fruto cruá vermelho (Sicana odorifera Naudin) e acompanhamento da estabilidade de seus compostos bioativos durante 0 armazenamento congelado
}

\author{
Characterization of musk cucumber (Sicana odorifera Naudin) \\ and monitoring of the stability of its bioactive compounds \\ during frozen storage
}

Jonathan Neves de Carvalho Alves ${ }^{1 *}$ (D), Victor Maximiliano Reis Tebaldi ${ }^{1}$, Kamila de Oliveira Nascimentoํㄹ ${ }^{2}$ Elisângela Elena Nunes Carvalho ${ }^{3}$, Rodrigo de Araújo Soares ${ }^{4}$, Ivanilda Maria Augusta ${ }^{5}$

${ }^{1}$ Centro Universitário de Barra Mansa, Departamento de Nutrição, Barra Mansa/RJ - Brasil ${ }^{2}$ Centro Universitário Geraldo Di Biase, Departamento de Nutrição, Volta Redonda/RJ - Brasil

${ }^{3}$ Universidade Federal de Lavras (UFLA), Departamento de Ciência dos Alimentos, Lavras/MG - Brasil

${ }^{4}$ Instituto Federal do Sertão Pernambucano, Ciência dos Alimentos, Petrolina/PE - Brasil

${ }^{5}$ Universidade Federal Rural do Rio de Janeiro (UFRRJ), Ciência e Tecnologia dos Alimentos, Seropédica/RJ Brasil

*Corresponding Author: Jonathan Neves de Carvalho Alves, Centro Universitário de Barra Mansa, Departamento de Nutrição, Rua Vereador Pinho de Carvalho, 267, CEP: 27330-550, Barra Mansa/RJ - Brasil, e-mail: jonathan.neves.alves@gmail.com

Cite as: Alves, J. N. C., Tebaldi, V. M. R., Nascimento, K. O., Carvalho, E. E. N., Soares, R. A., \& Augusta, I. M. (2021). Characterization of musk cucumber (Sicana odorifera Naudin) and monitoring of the stability of its bioactive compounds during frozen storage. Brazilian Journal of Food Technology, 24, e2020007.

https://doi.org/10.1590/1981-6723.00720

\begin{abstract}
Resumo
Cruá vermelho é um fruto aromático ainda pouco conhecido, originário da América Tropical, provavelmente do Brasil. É utilizado para fins alimentar, medicinal, repelente de insetos e aromatização de ambientes. Pouco se sabe acerca de suas características nutricionais. Diante do exposto, o presente trabalho visou realizar a caracterização física, físico-química e nutricional do fruto cruá vermelho. Os frutos foram caracterizados quanto a composição centesimal, pH, sólidos solúveis (SS), acidez titulável (AT), cálculo da ingestão diária recomendada (IDR), antocianinas, carotenoides totais, vitamina $\mathrm{C}$ e atividade antioxidante da polpa. A estabilidade dos carotenoides, da vitamina $\mathrm{C}$ e da cor dos frutos foi acompanhada durante 21 dias de armazenamento sob congelamento a $-18{ }^{\circ} \mathrm{C}$. Os carotenoides, a vitamina $\mathrm{C}$ e a cor do fruto foram afetados pelo congelamento durante o armazenamento. $\mathrm{O}$ fruto apresenta características apreciáveis do ponto de vista nutricional e funcional, uma vez que o consumo diário de $100 \mathrm{~g}$ de sua polpa fornece uma apreciável fração de nutrientes recomendados para ingestão diária por um indivíduo adulto. $\mathrm{O}$ fruto apresenta um baixo valor calórico, fato interessante para preparações dietéticas, além de apresentar capacidade antioxidante e compostos fitoquímicos de interesse para a saúde humana, como
\end{abstract}


carotenoides e antocianinas. Em relação à preservação dos compostos bioativos apresentados pelo fruto, verificouse que o congelamento não foi eficaz para a preservação de carotenoides e vitamina C. Diante de todas as características apresentadas, cruá vermelho se apresenta como uma excelente alternativa para inclusão na alimentação humana.

Palavras-chave: Fruto exótico; Cucurbitaceae; Melão-vermelho; Armazenamento sob congelamento; Estabilidade.

\begin{abstract}
Musk cucumber (Sicana odorifera Naudin) is a still little-known aromatic fruit native of Tropical America, probably Brazil. It is used as food and insect repellent, for medicinal purposes, and to flavor environments. Little is known about its nutritional characteristics. In this context, this study aimed to perform the physical, physicochemical and nutritional characterization of musk cucumber. The fruits were characterized according to their chemical composition, $\mathrm{pH}$, soluble solids (SS), titratable acidity (TA), recommended daily intake (RDI), anthocyanins, total carotenoids, vitamin C, and antioxidant activity of the pulp. The stability of carotenoids, vitamin C, and fruit color was monitored for 21 days of frozen storage $\left(-18^{\circ} \mathrm{C}\right)$. Carotenoids, vitamin $\mathrm{C}$, and fruit color were affected by freezing during storage. The fruit has appreciable characteristics from a nutritional and functional point of view, since the daily consumption of $100 \mathrm{~g}$ of its pulp provides a significant fraction of nutrients recommended for daily intake by an adult. The fruit presents low caloric value - an interesting fact for dietary preparations, in addition to having antioxidant capacity and phytochemicals of interest to human health, such as carotenoids and anthocyanins. Regarding the bioactive compounds present in the fruit, it was found that freezing was not effective to preserve carotenoids and vitamin C. Given all the characteristics presented, musk cucumber is an excellent alternative for inclusion in human diet.
\end{abstract}

Keywords: Exotic fruit; Cucurbitaceae; Red melon; Freezing storage; Stability.

\title{
1 Introdução
}

Cucurbitaceae é uma família botânica de trepadeiras amplamente cultivada em todo o mundo, representando importante relevância social, econômica e cultural (Fapohunda et al., 2018). Dentre as cucurbitáceas utilizadas pelo homem, encontram-se espécies cultivadas em virtude da qualidade sensorial e ornamental de seus frutos, e de suas características fitoterápicas (Souza et al., 2012; Parada et al., 2000).

A Sicana odorifera é uma espécie de cucurbitácea pouco conhecida, sendo uma trepadeira cujo comprimento pode atingir mais de 15 metros (Moreira, 1996; Morton, 1987). Seu fruto é popularmente conhecido como cruá vermelho, melão-de-cheiro, melão caroá, jamelão-de-caboclo e cassabanana. É um fruto cilíndrico de casca lisa, coloração que varia de vinho a roxo escuro, com cerca de $15 \mathrm{~cm}$ de diâmetro e $35 \mathrm{~cm}$ de comprimento (Madeira, 2016). A polpa é carnosa e amarelada, com sementes achatadas cor castanho-escuro, de aproximadamente $1 \mathrm{~cm}$ (Brasil, 2015). Apesar de sua ampla distribuição por toda América Tropical, acredita-se que esta espécie seja nativa do Brasil, mais especificamente do Recôncavo Baiano, e que tenha se disseminado por toda a América (Jesus et al., 2016; Kienteka et al., 2018; Rochelle, 1986).

Diversas são as utilidades do fruto cruá vermelho na cultura popular, dentre as quais, destaca-se sua utilização na culinária para preparações, como conservas, geleias, sucos e doces. Quando imaturo, pode ser consumido após a cocção, e após a maturação, é apreciado cru (Jaramillo et al., 2011; Rochelle, 1986; Kienteka et al., 2018). Além disso, o fruto pode ser armazenado por até seis meses após sua colheita, desde que não sofra injúrias (Priori et al., 2006).

Araújo (2016) produziu uma cerveja não convencional aproveitando a polpa do cruá vermelho, como adjunto do malte. O fruto auxiliou fornecendo minerais importantes para o processo fermentativo das 
leveduras. Um fato negativo foi que o produto ficou com a coloração escurecida, fato que não era esperado. Contudo, as análises sensoriais dos provadores do produto apontaram uma grande aceitação dele.

Acerca das características nutricionais de cruá vermelho, alguns nutrientes importantes, como as vitaminas A, C e E, algumas vitaminas do complexo B, além de minerais, como cálcio, potássio e fósforo, já foram identificados no fruto e se trata de nutrientes essenciais para a saúde humana (Filho et al., 2015). Todavia, poucas são as informações recentes sobre o aspecto nutricional do fruto. Alguns estudos já identificaram a presença de $\beta$-caroteno em cruá vermelho. Esse fitoquímico é responsável pela coloração amarelo alaranjada da polpa do fruto e, além de outras funções biológicas, desempenha um papel de precursor da vitamina A. Além disso, alguns trabalhos já foram realizados com o intuito de verificar alguns aspectos da composição físico-química do fruto, como composição centesimal, pH, acidez titulável (AT) e sólidos solúveis (SS), e a verificação da presença de alguns outros compostos bioativos (Filho et al., 2016; Reis et al., 2009; Morton, 1987; Araújo, 2016).

Diante da escassez de trabalhos recentes sobre o fruto cruá vermelho, o presente trabalho visou realizar sua caracterização física e físico-química, e composição nutricional, atividade antioxidante da polpa e acompanhamento da estabilidade da cor, dos carotenoides e da vitamina $\mathrm{C}$, durante 21 dias de armazenamento sob congelamento.

\section{Material e métodos}

\subsection{Coleta dos frutos e preparo da matéria-prima}

Os frutos cruá vermelho (S. odorifera), safra 2017, foram coletados de um espécime adulto cultivado no município de Barra Mansa-RJ (Latitude $22^{\circ} 32^{\prime} 39^{\prime}$ 'Sul, Longitude $44^{\circ} 10^{\prime} 17^{\prime \prime}$ Oeste). Os frutos encontravam-se no estádio inicial de maturação, com nove exemplares de boa qualidade visual e isentos de injúrias (Figura 1).

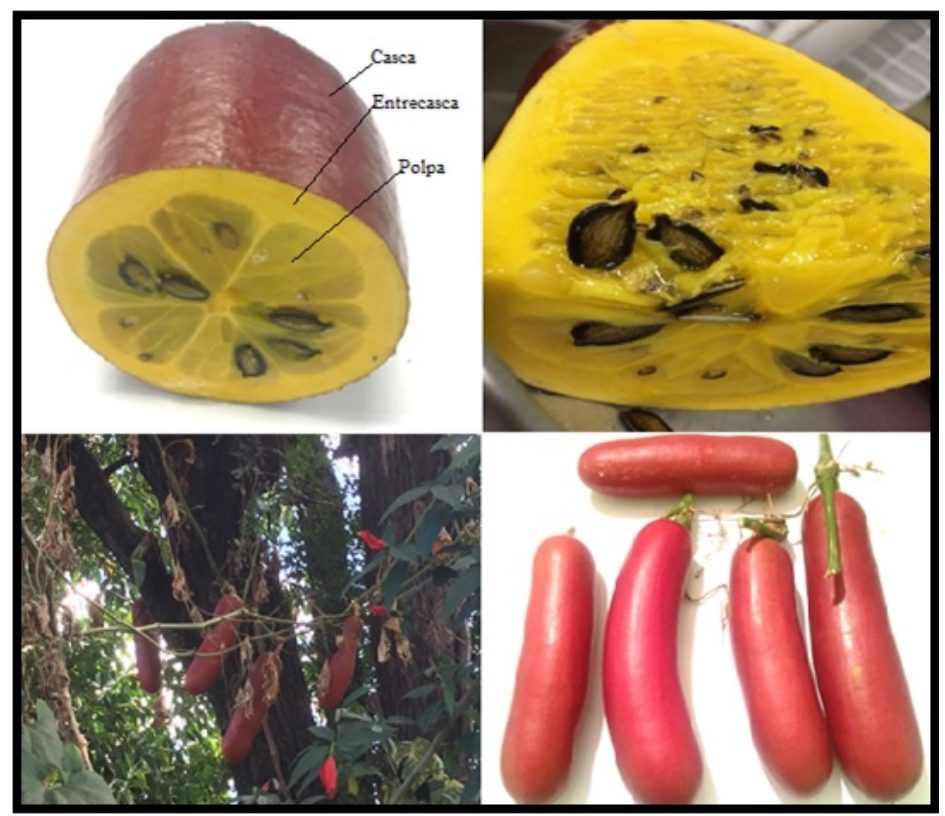

Figura 1. Frutos cruá vermelho (S. odorifera).

Após a colheita, os frutos foram imediatamente encaminhados ao Laboratório de Análises Bromatológicas do Centro Universitário de Barra Mansa (UBM), no qual foram higienizados e desmembrados em polpa (sem 
semente), casca e entrecasca. Cada parte foi macerada separadamente com o auxílio de um pistilo e grau, sendo todas armazenadas congeladas $\left(-18^{\circ} \mathrm{C}\right)$ até o momento das análises.

\subsection{Caracterização da matéria-prima}

A polpa do fruto foi submetida às análises de caracterização físico-química, capacidade antioxidante e determinação de compostos fenólicos no Instituto de Tecnologia de Alimentos do Departamento de Tecnologia de Alimentos da Universidade Federal Rural do Rio de Janeiro (UFRRJ). A polpa foi avaliada quanto à estabilidade de carotenoide e cor instrumental ao longo de 21 dias de armazenamento sob congelamento. Capacidade antioxidante, teor de compostos fenólicos e vitamina $\mathrm{C}$ foram determinados também na casca e na entrecasca do fruto.

\subsubsection{Determinação da composição físico-química da polpa e Ingestão Diária Recomendada (IDR)}

A composição centesimal da polpa foi determinada logo após a colheita, de acordo com os padrões da Association of Official Analytical Chemistry (2005): teor de umidade (Método 925.09); nitrogênio total (Método 2001.11, com fator de conversão de 5,75); gordura (Método 945.38), e cinzas (Método 923.03). A determinação de fibra bruta foi realizada de acordo com o método de digestão descrito pelo Instituto Adolfo Lutz (2008), enquanto o teor de carboidratos foi realizado por diferença.

O conteúdo de sólidos solúveis (SS) e a acidez titulável (AT) da polpa foram determinados seguindo as recomendações da Association of Official Analytical Chemistry (2005), com os resultados expressos em percentual (\%). A relação SS/AT foi realizada por meio do cálculo da razão destes dois valores. O potencial hidrogeniônico $(\mathrm{pH})$ foi obtido por leitura direta em peagâmetro digital Meter TEC-2® (Tecnal ${ }^{\circledR}$, São Paulo, SP, Brasil) (Association of Official Analytical Chemistry, 2012).

O cálculo da Ingestão Diária Recomendada (IDR) foi realizado de acordo com o Institute of Medicine (2005), tomando como referência a alimentação para um homem adulto com uma necessidade energética de $2.000 \mathrm{Kcal} / \mathrm{dia}$. O cálculo das necessidades de proteínas, carboidratos e lipídeos totais foi baseado na média aritmética de cada intervalo de recomendação. Utilizou-se também a relação de 4/4/9 para proteínas, carboidratos e lipídeos, respectivamente, para se obter o valor da IDR recomendada em grama de alimento. Todas as análises foram realizadas em triplicata.

\subsubsection{Capacidade antioxidante da polpa dos frutos}

A capacidade antioxidante foi determinada de acordo com o método DPPH, descrito por BrandWilliams et al. (1995) e Rufino et al. (2010). Foi preparada uma solução de metanol contendo 2,2-difenil-1picril-hidrazil (DPPH) $0,06 \mu \mathrm{M}$. Em seguida, adicionou-se uma alíquota de $100 \mu \mathrm{L}$ de extrato de amostras a $3,9 \mathrm{~mL}$ desta solução. A absorbância foi medida em espectrofotômetro UV (NI 2000 UV, Nova Instruments $\left.{ }^{\circledR}\right)$ a $517 \mathrm{~nm}$. A quantidade da capacidade antioxidante foi expressa em $\mu \mathrm{M}$ de trolox equivalente por $100 \mathrm{~g}$ de amostra (base úmida). A eliminação de radicais livres (\% SRL) de cada amostra foi calculada de acordo com a Equação 1.

$\% S R L=\frac{(\mathrm{Ac}-\mathrm{Aa}) \times 100}{A c}$

em que: Ac e Aa = valores de absorbância no controle e na amostra, respectivamente.

Toda a análise foi realizada em triplicata. 


\subsubsection{Determinação de compostos fenólicos totais e antocianinas}

Para a determinação dos compostos fenólicos da casca do fruto, extratos da casca foram obtidos de acordo com Swain \& Hillis (1959) e Torres et al. (2002) com modificações. Aproximadamente $1 \mathrm{~g}$ de amostra foi diluído em $50 \mathrm{~mL}$ de solução hidroalcoólica $70 \%$. Em seguida, a solução foi homogeneizada em agitador magnético a $25^{\circ} \mathrm{C}$ durante $1 \mathrm{~h}$ e, logo após, filtrada a vácuo. O conteúdo fenólico total da casca foi avaliado seguindo o ensaio de Folin-Ciocalteu descrito por Singleton et al. (1999) e Quettier-Deleu et al. (2000). Os resultados foram expressos em equivalentes de ácido gálico (GAE, $\mathrm{mg} / 100 \mathrm{~g}$ de massa fresca) utilizando uma curva padrão de ácido gálico $(0,05$ a $1,2 \mathrm{mg} / \mathrm{mL})$.

A casca do fruto cruá vermelho foi submetida à análise de antocianina. Para a extração de pigmentos de antocianinas nesses materiais, foi utilizada a solução de etanol 95\% acidificada com $\mathrm{HCl}$ 1,5 M (85:15 v/v), pH 1,0, obtendo a solução de extração segundo a metodologia de Fuleki \& Francis (1968). A leitura foi realizada em espectrofotômetro UV-visível (NI 2000 UV, Nova Instruments ${ }^{\circledR}$ ) em comprimento de onda de $520 \mathrm{~nm}$. Todas as análises foram feitas em triplicata.

\subsubsection{Determinação de carotenoides totais}

Para análise de carotenoides totais na polpa do fruto, foram preparados inicialmente extratos ricos em carotenoides com cada parte do fruto. Para obtenção dos extratos, pesou-se aproximadamente $0,5 \mathrm{~g}$ de amostras previamente trituradas diretamente em béquer $(50 \mathrm{~mL})$. O pigmento foi extraído da amostra com acetona $(10 \mathrm{~mL})$ e celite $(1 \mathrm{~g})$ utilizando almofariz e pistilo de porcelana, sendo as amostras filtradas em funil com placa de vidro sinterizado até que o resíduo se apresentasse destituído de cor (quatro extrações).

Em um funil de separação contendo aproximadamente $50 \mathrm{~mL}$ de éter de petróleo, foi adicionado o extrato de carotenoides (obtido da extração), seguido de adição cuidadosa de água destilada (aproximadamente $300 \mathrm{~mL}$ ). Após a separação das fases, a fase aquosa, constituída de água e acetona, foi descartada. O procedimento foi repetido até que todo o extrato fosse transferido para o éter de petróleo.

Posteriormente, a fase etérea foi lavada cinco vezes com água destilada para a remoção completa de acetona. Após o descarte da água da última lavagem, o extrato etéreo foi recolhido num balão volumétrico $(50 \mathrm{~mL})$ fazendo-o passar por funil de vidro contendo uma pequena porção de sulfato de sódio anidro $(2 \mathrm{~g})$ para remoção de eventual água residual, sendo recolhido em um balão volumétrico. Após o ajuste do volume, procedeu-se à leitura da absorbância em comprimento de $450 \mathrm{~nm}$ em espectrofotômetro UV-Visível (Rodriguez-Amaya, 2001).

O valor de absortividade dos carotenoides (A1\% $1 \mathrm{~cm}$ ) foi calculado de acordo com a Equação 2 e expresso em $\mu \mathrm{g} / \mathrm{g}$.

$$
C\left(\mu \frac{g}{g}\right)=\frac{\text { Absorbâcia } \times \text { volume }(\mathrm{mL}) 10^{4}}{(\text { A } 1 \% 1 \mathrm{~cm} \times \text { peso da amostra }(\mathrm{g})}
$$

\subsubsection{Determinação da vitamina C}

A determinação de vitamina $\mathrm{C}$ (ácido ascórbico) foi realizada conforme o método descrito pelo Instituto Adolfo Lutz (2008), no qual, por meio de titulação com iodato de potássio, pode-se calcular o volume gasto de reagente. Em seguida, multiplicou-se o produto correspondente ao volume de reagente gasto pelo fator do ácido ascórbico, que é 0,8806 , e subtraiu-se do peso das amostras. 


\subsubsection{Estudo da estabilidade de carotenoides totais e vitamina $C$ da polpa e da entrecasca do fruto durante congelamento}

O conteúdo de carotenoides totais na polpa de cruá vermelho e de vitamina $\mathrm{C}$ em sua polpa e entrecasca foi determinado imediatamente após a colheita do fruto. A evolução da estabilidade de carotenoides e vitamina $\mathrm{C}$ foi determinada em quatro pontos distintos, durante 21 dias de armazenamento congelado $\left(-18{ }^{\circ} \mathrm{C}\right)$. As análises foram realizadas em triplicatas.

\subsubsection{Determinação da cor instrumental da polpa dos frutos cruá vermelho}

A análise da cor da polpa dos frutos congelada e armazenada por até 21 dias foi realizada utilizando colorímetro portátil (CR-410, Minolta Sensing Konica, Inc., Tóquio, Japão). As coordenadas $L^{*}, a^{*}$ e $b^{*}$ foram obtidas por meio da escala da Comissão Internacional de Iluminação (CIE), utilizando o iluminante D65 e observador em $10^{\circ}$. O ângulo de tonalidade $(h)$ e o croma $\left(C^{*}\right)$ foram calculados a partir das equações propostas por Balthazar et al. (2015).

\subsection{Análise estatística}

O experimento foi conduzido em um delineamento inteiramente casualizado (DIC), com três repetições. Os resultados das análises físico-químicas foram avaliados por meio de cálculos de média e desvio padrão.

$\mathrm{O}$ acompanhamento da estabilidade da cor, de compostos carotenoides e da vitamina $\mathrm{C}$ foi analisado por meio da ANOVA. As médias foram comparadas pelo teste de Tukey a 5\% de probabilidade, utilizando o software estatístico SISVAR (Ferreira, 2014), e os resultados foram obtidos por meio da análise de regressão.

\section{Resultados e discussão}

\subsection{Caracterização físico-química}

A caracterização físico-química do fruto in natura pode ser verificada na Tabela 1, bem como seu percentual de representatividade na Ingestão Diária Recomendada (IDR) para um indivíduo adulto com uma simulada demanda energética de $2.000 \mathrm{kcal}$ e possuindo planejamento alimentar composto por $65 \%$ de carboidratos, $20 \%$ de lipídeos e $15 \%$ de proteínas. Além disso, com uma demanda de $25 \mathrm{~g}$ de fibras alimentares por dia.

Tabela 1. Composição físico-química do fruto cruá vermelho (S. odorifera).

\begin{tabular}{ccc}
\hline Parâmetros & Cruá vermelho $(\mathbf{g} / \mathbf{1 0 0} \mathbf{g})$ & \%IDR (Institute of Medicine, 2005) \\
\hline Umidade & $83,33 \pm 0,43$ & ND \\
\hline Proteína & $4,93 \pm 0,57$ & $6,6 \%$ \\
\hline Lipídios & $0,91 \pm 0,06$ & $2,0 \%$ \\
\hline Cinzas & $4,63 \pm 0,14$ & $\mathrm{ND}$ \\
\hline Fibra bruta & $1,10 \pm 0,28$ & $3,7 \%$ \\
\hline Carboidratos & $5,10 \pm 0,41$ & $1,6 \%$ \\
\hline V.e.t. $(k c a l)$ & $48,31 \pm 0,13$ & $2,4 \%$ \\
\hline $\mathrm{pH}$ & $6,00 \pm 0,10$ & $\mathrm{ND}$ \\
\hline Acidez titulável (\% ácido cítrico) & $0,12 \pm 0,03$ & $\mathrm{ND}$ \\
\hline$\%$ Sólidos solúveis $\left({ }^{\circ}\right.$ Brix) & $16 \pm 1,00$ & $\mathrm{ND}$ \\
\hline
\end{tabular}

Média \pm DP = intervalo confiável para uma probabilidade estatística de 95\%; os resultados apresentados na tabela correspondem à base úmida. Cada valor é apresentado como média \pm desvio padrão $(\mathrm{n}=3)$. IDR = Ingestão Diária Recomendada; Ve.t. = Valor Energético Total: Kcal (quilocalorias); ND = Não determinado. 
Observou-se um elevado teor de umidade, semelhantemente aos teores encontrados em frutos de Citrullus lanatus (92,3\%) e Cucumis melo (93,3\%), ambos da família Cucurbitaceae (Morais et al., 2017). O teor de umidade dos frutos está relacionado com sua perecibilidade (Coutinho \& Cantillano, 2007).

$\mathrm{O}$ presente estudo encontrou um percentual proteico considerável na polpa do fruto, sendo $100 \mathrm{~g}$ correspondentes a aproximadamente $6,6 \%$ das necessidades proteicas diárias de um indivíduo adulto. Devese levar em consideração o fato de se tratar de um alimento de origem vegetal, que naturalmente exibe quantidades reduzidas desse importante macronutriente, o que valoriza os dados do conteúdo proteico do fruto aqui estudado. O conteúdo proteico do fruto cruá vermelho é superior aos encontrados em frutos da mesma família, como a Citrullus lanatus (0,9\%), o Cucumis melo (0,7\%) e o Cucumis sativus $(0,9 \%)$ (Universidade Estadual de Campinas, 2011).

Acerca das fibras, o presente estudo encontrou um teor semelhante ao encontrado em mamão papaia (Carica papaya L.), que foi de 1,8\% (Morais et al., 2017). O interesse pelas fibras alimentares se deve a seus diversos efeitos positivos à saúde, como a regulação intestinal e a redução dos níveis de glicose e lipídeos séricos (Mineiro, 2014).

A relação sólidos solúveis/acidez titulável da polpa do fruto cruá vermelho foi de 133,33, evidenciando que o fruto foi colhido maduro. Os sólidos solúveis estão relacionados com a aceitação do gênero alimentício, devido à sua relação com a sua doçura (Silva et al., 2002).

Em relação ao $\mathrm{pH}$, o valor encontrado $(6,00)$ indica que a polpa é levemente ácida, semelhante aos valores encontrados por outros autores (Tabela 2).

Na Tabela 2, estão expressos os valores de análises físico-químicas do fruto cruá vermelho em seu estado in natura, disponíveis na literatura científica.

Tabela 2. Comparação entre composições físico-químicas em fruto cruá vermelho (S. odorifera).

\begin{tabular}{cccccc}
\hline Parâmetros & $\begin{array}{c}\text { Morton } \\
(\mathbf{1 9 8 7})\end{array}$ & $\begin{array}{c}\text { Reis et al. } \\
\mathbf{( 2 0 0 9 )}\end{array}$ & $\begin{array}{c}\text { Filho et al. } \\
\mathbf{( 2 0 1 5 )}\end{array}$ & $\begin{array}{c}\text { Araújo } \\
\mathbf{( 2 0 1 6 )}\end{array}$ & $\begin{array}{c}\text { Presente } \\
\text { trabalho }\end{array}$ \\
\hline Umidade & 85,10 & 90,40 & 82,46 & 85,20 & 83,33 \\
\hline Proteína & 0,14 & 0,91 & 2,62 & 1,09 & 4,93 \\
\hline Lipídios & 0,02 & 0,22 & 1,00 & 0,35 & 0,91 \\
\hline Cinzas & 0,70 & 1,06 & 0,77 & ND & 4,63 \\
\hline Fibra & 1,10 & ND & 1,33 & ND & 1,10 \\
\hline Carboidratos & ND & 7,41 & 11,81 & 10,54 & 5,10 \\
\hline VET $(\mathrm{kcal})$ & ND & 35,26 & 66,77 & ND & 48,31 \\
\hline pH & ND & ND & 6,61 & 6,20 & 6,00 \\
\hline AT & ND & ND & 0,33 & 0,20 & 0,12 \\
\hline SS $\left({ }^{\circ}\right.$ Brix $)$ & ND & ND & 4,15 & 12,10 & 16 \\
\hline
\end{tabular}

$\mathrm{ND}=$ Não determinado; $\mathrm{SS}\left({ }^{\circ}\right.$ Brix $)=$ Sólidos Solúveis; AT = Acidez titulável em ácido cítrico.

O estado de maturação do fruto em estádios diferentes, ou ainda, as condições ambientais do local de colheita do fruto podem influenciar em suas características físico-químicas (Nascimento et al., 2014).

\subsection{Compostos fenólicos, antocianinas e capacidade antioxidante}

Na Tabela 3, observam-se os valores dos compostos fenólicos e antocianinas totais em cruá vermelho, bem como seu percentual de sequestro de radicais livres (\%SRL) e, finalmente, sua capacidade antioxidante. 
Tabela 3. Compostos fenólicos e a capacidade antioxidante do fruto cruá vermelho (S. odorifera).

\begin{tabular}{cc}
\hline Fenólicos (mg de ácido gálico/100 g de amostra de casca) & $691,41 \pm 4,60$ \\
\hline Capacidade antioxidante $(\mu \mathrm{M}$ eq. trolox/g de amostra de polpa) & $1957,58 \pm 0,69$ \\
\hline \%SRL (polpa) & $78,13 \pm 4,42$ \\
\hline Antocianinas totais $(\mathrm{mg} / 100 \mathrm{~g}$ de amostra de casca) & $04,15 \pm 0,42$ \\
\hline Carotenoides totais $(\mu \mathrm{g} / \mathrm{g}$ de polpa) & $36,92 \pm 0,67$ \\
\hline Vitamina C $(\mathrm{mg} / 100 \mathrm{~g}$ de polpa) & $32,60 \pm 4,81$ \\
\hline Vitamina C $(\mathrm{mg} / 100 \mathrm{~g}$ de entrecasca) & $16,71 \pm 2,90$ \\
\hline
\end{tabular}

Média \pm DP = intervalo confiável para uma probabilidade estatística de $95 \%$; cada valor é apresentado como média \pm desvio padrão $(n=3)$; $\mathrm{SRL}=$ Sequestro de Radicais Livres; Eq. = Equivalente; $\mu \mathrm{g}=$ micrograma; $\mu \mathrm{M}=$ micromolar.

O fruto cruá vermelho apresentou valores interessantes de compostos fenólicos. Para fins de comparação com um fruto da mesma família botânica, Tamer et al. (2010) encontraram 476,6 mg/100 g de polpa de compostos fenólicos em Cucurbita moschata L, teores inferiores aos encontrados em cruá vermelho. Os compostos fenólicos são grupos variados de produtos do metabolismo secundário dos vegetais e apresentam alta capacidade antioxidante (Dias et al., 2015). Segundo Kuskoski et al. (2006) e Melo et al. (2008), os compostos fenólicos são os principais responsáveis pela capacidade antioxidante em frutos, sendo responsáveis por mais de $90 \%$ da capacidade antioxidante total de frutos. Segundo Brandão et al. (2011), o processo de maturação reduz a quantidade de compostos fenólicos em frutos; desta forma, acredita-se que os frutos cruá vermelho imaturos apresentem quantidades superiores às encontradas no presente trabalho.

Já as antocianinas são substâncias com diversas funções. Estas substâncias fenólicas conferem cor e realizam atividades biológicas importantes, como a função antioxidante, anti-inflamatória, antimicrobiana e até mesmo a função neuroprotetora (Khoo et al., 2017).

O conteúdo de antocianinas na casca do fruto cruá vermelho (Tabela 3) encontrado no presente trabalho foi inferior ao conteúdo de antocianinas de outros frutos, como é o caso da amora (Morus), que apresenta $201 \mathrm{mg} / 100 \mathrm{~g}$; do mirtilo (Vaccinium myrtillus), com 243,8 mg/100 g, e da jabuticaba (Plinia cauliflora), que apresenta cerca de $48 \mathrm{mg} / 100 \mathrm{~g}$. Esses frutos mencionados são reconhecidos por conterem uma alta concentração de antocianinas (Rigolon, 2017).

Melo et al. (2008) classificaram o \%SRL de extratos da polpa de diversos frutos, incluindo várias espécies da família Cucurbitaceae, da seguinte maneira: baixo, quando abaixo de $50 \%$; moderado, quando entre $50 \%$ e 70\%, e elevado, quando acima de 70\%. Levando-se em consideração a classificação proposta pelos autores, o fruto cruá vermelho exibiu um elevado percentual de sequestro de radicais livres $(78,13 \%)$.

A polpa do fruto cruá vermelho exibiu alta capacidade antioxidante $(1.957,58 \mu \mathrm{M}$ eq. trolox/g) quando comparada à atividade antioxidante de outros frutos, como é o caso, por exemplo, dos resultados encontrados por Gonçalves (2008), o qual analisou o extrato de frutos de camu-camu (Myrciaria dubia), que já é bem estabelecido como um fruto com alta atividade antioxidante ( $1.439 \mu \mathrm{M}$ eq. trolox $/ \mathrm{g}) ;$ abiu (Pouteria caimito) $(69 \mu \mathrm{M}$ eq. trolox $/ \mathrm{g})$; tamarindo (Tamarindus indica) $(21 \mu \mathrm{M}$ eq. trolox $/ \mathrm{g})$, entre outros resultados. Já Genovese et al. (2008) e Hoffmann-Ribani et al. (2009) encontraram, em morango (Fragaria $\times$ ananassa), $1.221 \mu \mathrm{M}$ eq. trolox/g. Todos estes trabalhos utilizaram o mesmo método de análise que o presente trabalho. Diante disso, o poder antioxidante do fruto cruá vermelho pode ser considerado elevado.

\subsection{Estabilidade de caronetóides totais durante o armazenamento do fruto}

Os carotenoides apresentam uma importante capacidade antioxidante. Essa capacidade se deve às suas duplas ligações conjugadas, as quais são susceptíveis à oxidação quando expostas à luz e/ou ao oxigênio. Devido a isso, os carotenoides têm a capacidade de sequestrar o oxigênio singleto e reagir com radicais livres. Além das atuações no organismo já citadas, os carotenoides totais podem desempenhar diversas outras 
funções de interesse, como atuação no sistema imunológico, ação precursora da vitamina A, entre outras (Kobori, 2010).

O tempo de armazenamento da polpa do fruto congelado influenciou significativamente no teor de carotenoides totais do fruto cruá vermelho. Na Figura 2, nota-se que houve perda significativa de compostos carotenoides entre os tempos 0 e 7 dias, e entre os tempos 14 e 21 dias, perdas as quais foram consideradas estatisticamente significativas. Entre os tempos 7 e 14 dias, não houve diferença significativa. Segundo Ribeiro et al. (2018), devido à sua estrutura com uma longa cadeia altamente insaturada, ocorrem reações que resultam em uma instabilidade nas estruturas dos carotenoides durante o armazenamento e o processamento dos alimentos. Os carotenoides podem sofrer isomerização geométrica e oxidação (decomposição) resultante da exposição à luz e ao oxigênio, da elevação de temperatura e da presença de oxigênio singlete e de radicais livres. Tudo isso pode culminar em uma perda de seu poder corante e redução de sua atividade antioxidante e pró-vitamínica.

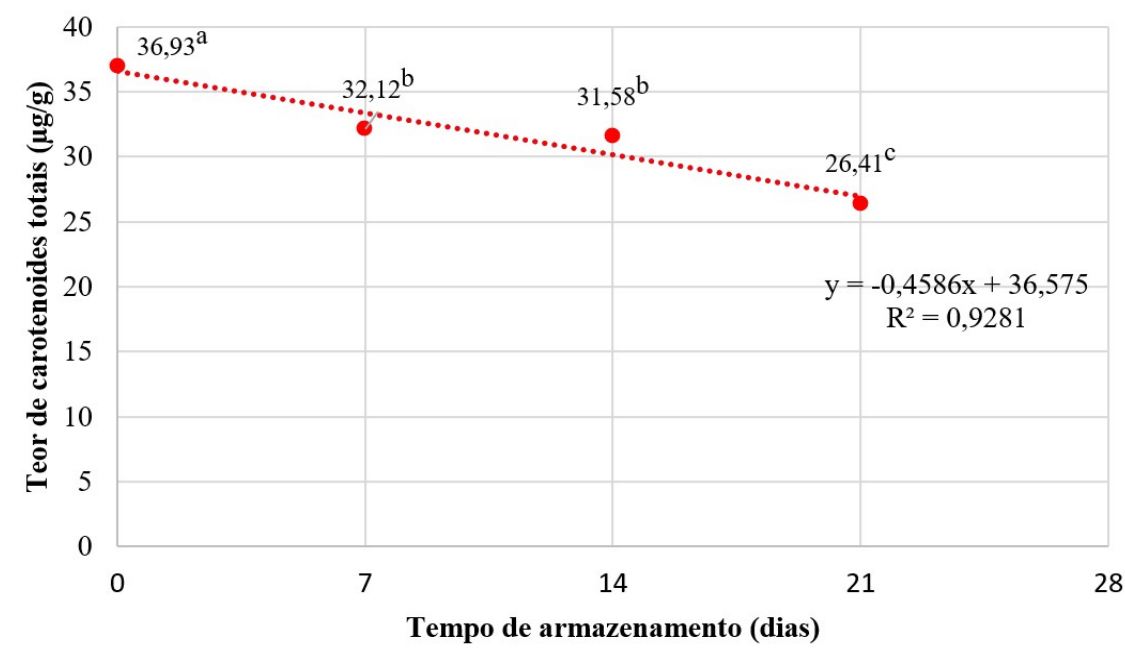

Figura 2. Teor médio de carotenoides totais da polpa do fruto cruá vermelho (S. odorifera) durante 21 dias de armazenamento sob congelamento.

Os carotenoides são os principais responsáveis pela coloração, que varia entre o amarelo, passando pelo laranja e vermelho, nos alimentos de origem vegetal. Eles também têm sido amplamente utilizados na indústria alimentícia como corantes naturais, tendo como objetivo fornecer cor aos produtos alimentícios e reparar a coloração perdida durante os processos de armazenamento e processamento de alimentos de origem vegetal (Lima et al., 2010).

\subsection{Estabilidade de vitamina C durante o armazenamento do fruto}

$\mathrm{O}$ teor de vitamina $\mathrm{C}$ na polpa do fruto cruá vermelho foi de $32,6 \mathrm{mg} / 100 \mathrm{~g}$. Considerando-se as referências atuais (Institute of Medicine, 2005), as quais preconizam uma ingestão diária de $75 \mathrm{mg} / 100 \mathrm{~g} \mathrm{e} 90 \mathrm{mg} / 100 \mathrm{~g}$ para homens e mulheres, respectivamente, o consumo de $100 \mathrm{~g}$ de polpa do fruto cruá vermelho atende a $43,5 \%$ e $36,2 \%$ para mulheres e homens, respectivamente, das recomendações de ingestão diária desse importante micronutriente. Para fins de comparação, uma laranja lima (Citrus), em seu estado in natura, apresenta cerca de $43,5 \mathrm{mg} / 100 \mathrm{~g}$, evidenciando que o cruá vermelho também se mostra uma boa opção para consumo, uma vez que $200 \mathrm{~g}$ de seu consumo atendem quase que, na integridade, a IDR para um adulto. A entrecasca do fruto também exibiu bom conteúdo de vitamina $\mathrm{C}$, porém inferior ao presente na polpa do fruto. A entrecasca atende a $22,3 \%$ e $18,5 \%$ da IDR para mulheres e homens, respectivamente. 
Houve um decréscimo significativo no teor de vitamina $\mathrm{C}$ do fruto durante os 21 dias de armazenamento congelado, sendo que esse fenômeno ocorreu tanto na polpa (redução de 87,4\%) quanto na entrecasca (redução de 96,2\%). Segundo Sucupira et al. (2012), a vitamina C é o micronutriente que mais se degrada quimicamente em alimentos, pois ela pode ter seus teores reduzidos por diversos fatores, como $\mathrm{pH}$, oxigênio, luz, temperatura, teor de umidade ou atividade de água. Conforme observado na Figura 3, houve diferença significativa $(p<0,05)$ entre os tempos de armazenamento e entre os tratamentos (polpa e entrecasca). A redução de vitamina $\mathrm{C}$ para a polpa pode ser equacionada de acordo com a função polinomial de $2 .^{\circ}$ grau (3), já a sua redução na entrecasca ocorre segundo a Equação linear (4).

$$
\begin{aligned}
& y=0,0413 \times 2-2,0987 x+31,274(\mathrm{R} 2=0,9168) \\
& y=-0,7306 \mathrm{x}+14,836(\mathrm{R} 2=0,9182)
\end{aligned}
$$

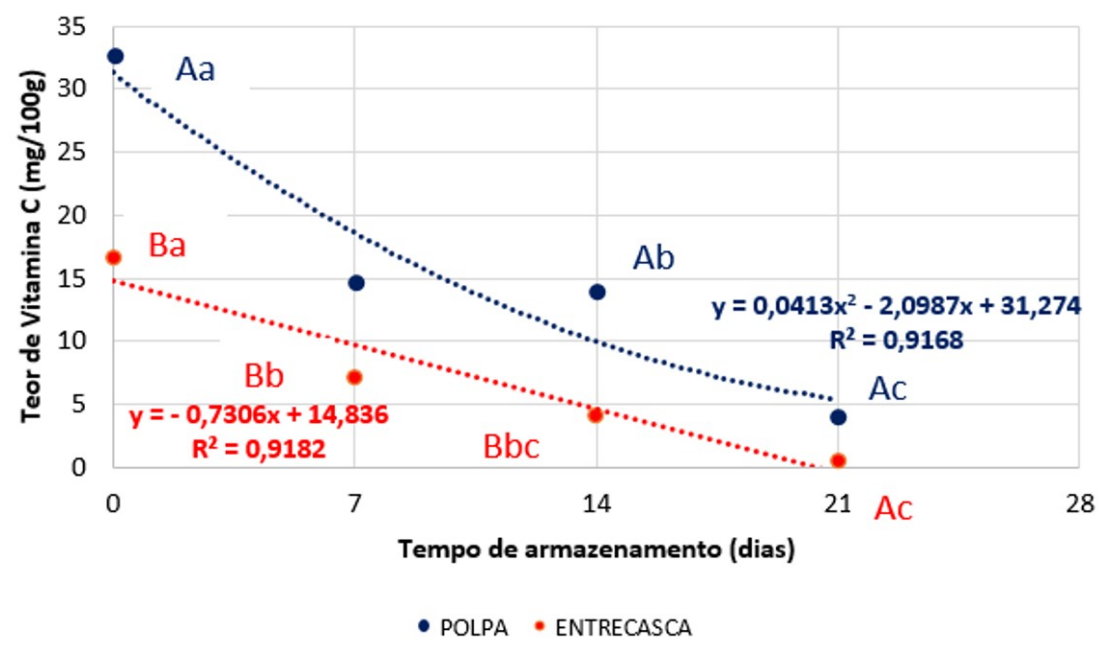

Figura 3. Teor médio de vitamina $\mathrm{C}$ da polpa e da entrecasca do fruto cruá vermelho ( $S$. odorifera) durante 21 dias de armazenamento sob congelamento.

\subsection{Análise de cor}

O resultado das análises de cor durante o armazenamento congelado $\left(-18{ }^{\circ} \mathrm{C}\right)$ da polpa do fruto cruá vermelho pode ser observado na Tabela 4.

Tabela 4. Valores médios ${ }^{\dagger}$ para os parâmetros de cor da polpa de cruá vermelho (S. odorifera).

\begin{tabular}{ccccc}
\hline \multirow{2}{*}{ Parâmetros } & $\mathbf{4}$ & \multicolumn{3}{c}{ Tempo } \\
\cline { 2 - 5 } & $\mathbf{0}$ & $\mathbf{7}$ & $\mathbf{1 4}$ & $\mathbf{2 1}$ \\
\hline $\mathrm{L}^{*}$ & $63,75 \pm 0,76^{\mathrm{a}}$ & $62,93 \pm 0,60^{\mathrm{ab}}$ & $62,46 \pm 1,08^{\mathrm{b}}$ & $62,46 \pm 0,32^{\mathrm{b}}$ \\
\hline $\mathrm{a}^{*}$ & $13,55 \pm 0,29^{\mathrm{a}}$ & $13,47 \pm 0,05^{\mathrm{a}}$ & $13,28 \pm 0,52^{\mathrm{a}}$ & $13,28 \pm 0,22^{\mathrm{a}}$ \\
\hline $\mathrm{b}^{*}$ & $62,85 \pm 0,90^{\mathrm{a}}$ & $63,49 \pm 0,46^{\mathrm{a}}$ & $62,18 \pm 2,57^{\mathrm{a}}$ & $62,18 \pm 0,48^{\mathrm{a}}$ \\
\hline $\mathrm{C}^{*}$ & $64,29 \pm 0,11^{\mathrm{b}}$ & $64,90 \pm 0,28^{\mathrm{a}}$ & $63,58 \pm 0,41^{\mathrm{c}}$ & $63,58 \pm 0,17^{\mathrm{c}}$ \\
\hline $\mathrm{h}^{*}$ & $4,63 \pm 0,23^{\mathrm{a}}$ & $4,71 \pm 0,17^{\mathrm{a}}$ & $4,68 \pm 0,34^{\mathrm{a}}$ & $4,68 \pm 0,13^{\mathrm{a}}$ \\
\hline
\end{tabular}

${ }^{\dagger}$ Médias \pm desvio padrão de nove observações (três repetições; três replicatas). ${ }^{\text {a-c }}$ Médias seguidas por diferentes letras na linha diferem significativamente $(p<0,05)$ pelo teste de Tukey a $5 \%$ de significância.

O parâmetro L* indica luminosidade, $\mathrm{C}^{*}$ representa a saturação e $\mathrm{h}$ é o ângulo de tonalidade. $\mathrm{O}$ valor de saturação $C^{*}$ representa a distância do eixo de luminosidade $\left(L^{*}\right)$ e inicia em zero no centro. $O$ ângulo de 
tonalidade começa no eixo $+\mathrm{a}^{*}$ e se movimenta em sentido anti-horário. É expresso em graus (por exemplo, $0^{\circ}$ é vermelho e $90^{\circ}$ é amarelo).

Observou-se uma ligeira variação no parâmetro $a^{*}[(-)$ verde / (+) vermelho]. Observa-se, também, variação nos pigmentos (-) azul / amarelo $(+)$, representados pelo parâmetro $b^{*}$. Com relação ao croma $\left(C^{*}\right)$, quanto mais altos os valores, mais viva a cor observada; quanto mais baixo o valor, mais opaca a mesma. Também houve uma variação no parâmetro croma durante o período de armazenamento congelado.

Segundo Harder et al. (2007), a pigmentação de um alimento também é influenciada pelo seu teor de carotenoides. Os dados apresentados na Tabela 4 sugerem que houve uma ligeira variação na pigmentação da polpa congelada do fruto cruá vermelho. Todavia, não se observou diferença estatística em todos os parâmetros, apenas nos parâmetros $b^{*} \mathrm{e} c^{*}$. De forma geral, a degradação de carotenoides e a possível degradação de outros pigmentos justificam a alteração na coloração do fruto.

\section{Conclusão}

O fruto cruá vermelho possui características apreciáveis do ponto de vista funcional, tanto em sua polpa, quanto na entrecasca; além disso, o fruto apresenta um baixo valor calórico, que o torna de interesse em aplicações dietéticas. $\mathrm{O}$ fruto exibe boa capacidade antioxidante quando comparado a outros frutos $\mathrm{e}$ apresenta compostos bioativos de interesse para a saúde humana, como carotenoides e antocianinas.

Nesse trabalho, o congelamento não exibe eficiência na preservação de carotenoides e vitamina C. Diante disso, sugere-se que métodos de preservação de vitamina $\mathrm{C}$ e carotenoides sejam aplicados durante o processamento e o armazenamento do fruto, uma vez que reduções extremas destes compostos não são interessantes do ponto de vista nutricional e comercial.

Diante de todas as características apresentadas, cruá vermelho se apresenta como uma excelente alternativa para inclusão na alimentação humana.

\section{Referências}

Araújo, G. S. (2016). Elaboração de uma cerveja ale utilizando melão de caroá [Sicana odorifera (Vell.) Naudim] como adjunto do malte (Dissertação de mestrado). Programa de Pós-graduação em Engenharia Química, Universidade Federal da Bahia, Salvador.

Association of Official Analytical Chemistry - AOAC. (2005). Official methods of analysis of the association of Official Analytical Chemistry (18th ed.) Gaithersburg: AOAC international. Recuperado em 16 de dezembro de 2019, de https://www.researchgate.net/publication/292783651_AOAC_2005

Association of Official Analytical Chemistry - AOAC. (2012). Official methods of analysis of the Association of Official Analytical Chemistry. Washington: AOAC international.

Balthazar, C. F., Silva, H. L., Celeguini, R. M., Santos, R., Pastore, G. M., Junior, C. A., Freitas, M. Q., Nogueira, L. C., Silva, M. C., \& Cruz, A. G. (2015). Effect of galactooligosaccharide addition on the physical, optical, and sensory acceptance of vanilla ice cream. Journal of Dairy Science, 98(7), 4266-4272. PMid:25912870. http://dx.doi.org/10.3168/jds.2014-9018

Brandão, T. S. O., Sena, A. R., Teshima, E., David, J. M., \& Assis, S. A. (2011). Changes in enzymes, phenolic compounds, tannins, and vitamin $\mathrm{C}$ in various stages of jambolan (Syzygium cumini Lamark) development. Food Science and Technology, 31(4), 849-855. http://dx.doi.org/10.1590/S0101-20612011000400004

Brand-Williams, W., Cuvelier, M. E., \& Berset, C. (1995). Use of a free radical method to evaluate antioxidant activity. Lebensmittel-Wissenschaft + Technologie, 28(1), 25-30. http://dx.doi.org/10.1016/S0023-6438(95)80008-5

Brasil. Ministério da Saúde. Secretaria de Atenção à Saúde. Departamento de Atenção Básica. (2015). Alimentos regionais brasileiros (2. ed.). Brasília. Recuperado em 16 de dezembro de 2019, de http://bvsms.saude.gov.br/bvs/publicacoes/alimentos_regionais_brasileiros_2ed.pdf

Coutinho, E. F., \& Cantillano, F. F. (2007). Sistema de produção do mirtilo. Pelotas: Embrapa Clima Temperado. Versão eletrônica. Recuperado em 16 de dezembro de 2019, de https://www.infoteca.cnptia.embrapa.br/bitstream/doc/745223/1/sistema08.pdf

Dias, T., Melo, H. C., Alves, F. R. R., Carvalho, R. F., Carneiro, K. S., \& Sousa, C. M. (2015). Compostos fenólicos e capacidade antioxidante em frutos de tomateiros mutantes fotomorfogenéticos. Ciência Rural, 45(5), 782-787. http://dx.doi.org/10.1590/0103-8478cr20140098 
Fapohunda, S. O., Aderiike, A. A., \& David, O. J. (2018). Cucurbitaceae: The family that nourishes and heals. Micro Medicine., 6(2), 85-93. http://dx.doi.org/10.5281/zenodo.1436798

Ferreira, D. F. (2014). Sisvar: a Guide for its Bootstrap procedures in multiple comparisons. Ciência e Agrotecnologia, 38(2), 109-112. http://dx.doi.org/10.1590/S1413-70542014000200001

Filho, P. G. X., Barreira, T. F., Pinheiro, S. S., Morais Cardoso, L., Duarte Martino, H. S., \& Pinheiro-Sant'Ana, H. M. (2015). Melão croá' (Sicana sphaerica Vell.) and 'maracujina' (Sicana odorifera Naud.): chemical composition, carotenoids, vitamins. Fruits, 70(6), 341-349. http://dx.doi.org/10.1051/fruits/2015035

Fuleki, T., \& Francis, F. J. (1968). Quantitative methods for anthocyanins: 1. Extraction and determination of total anthocyanin in cranberries. Journal of Food Science, 33(1), 72-77. http://dx.doi.org/10.1111/j.1365-2621.1968.tb00887.x

Genovese, M. I., Pinto, M. S., Gonçalves, A. E. S. S., \& Lajolo, F. M. (2008). Bioactive compounds and antioidant capacity of exotiic fruids and comercial frozen pulps fron Brazil. Food Science \& Technology International, 14(3), 207-214. http://dx.doi.org/10.1177/1082013208092151

Gonçalves, A. E. S. S. (2008). Avaliação da capacidade antioxidante de frutas e polpas de frutas nativas e determinação dos teores de flavonoides e vitamina C (Dissertação de mestrado). Universidade de São Paulo, São Paulo.

Harder, M. N. C., Canniatti-Brazaca, S. G., \& Arthur, V. (2007). Avaliação quantitativa por colorímetro digital da cor do ovo de galinhas poedeiras alimentadas com urucum (Bixa orellana). Revista Portuguesa de Ciências Veterinárias, 102(563-564), 339342.

Hoffmann-Ribani, R., Huber, L. S., \& Rodriguez-Amaya, D. B. (2009). Flavonols in fresh and processed Brazilian fruits. Journal of Food Composition and Analysis, 22(4), 263-268. http://dx.doi.org/10.1016/j.jfca.2008.12.004

Institute of Medicine - IOM. (2005). Dietary reference intakes: Energy, carbohydrate, fiber, fat, fatty acids, cholesterol, protein, and amino acids. Washington, D.C.: National Academies Press. https://doi.org/10.17226/10490.

Instituto Adolfo Lutz - IAL. (2008). Normas analíticas: métodos químicos e físicos para análise de alimentos (1020 p.). São Paulo: IAL. Recuperado em 16 de dezembro de 2019, de

http://www.ial.sp.gov.br/resources/editorinplace/ial/2016_3_19/analisedealimentosial_2008.pdf

Jaramillo, K., Dawid, C., Hofmann, T., Fujimoto, Y., \& Osorio, C. (2011). Identification of antioxidative flavonols and anthocyanins in Sicana odorifera fruit peel. Journal of Agricultural and Food Chemistry, 59(3), 975-983. PMid:21244058. http://dx.doi.org/10.1021/jf103151n

Jesus, G. F., Bastos, J. S., Oliveira, N. A., Anjos, M. B., Santos, J. C. R., Souza, S. M. A., \& Martinez, E. A. (2016). Estudos preliminares na formulação de estruturados de mix de polpa de frutas. Revista do Congresso Sul Brasileiro de Engenharia de Alimentos, 2(1), 10

Khoo, H. E., Azlan, A., Tang, S. T., \& Lim, S. M. (2017). Anthocyanidins and anthocyanins: colored pigments as food, pharmaceutical ingredients, and the potential health benefits. Food \& Nutrition Research, 61(1), 1361779. PMid:28970777. http://dx.doi.org/10.1080/16546628.2017.1361779

Kienteka, S. S., Corrêa-Ferreira, M. L., \& de Oliveira Petkowicz, C. L. (2018). Characterization of cell wall polysaccharides from Sicana odorifera fruit and structural analysis of a galactan-rich fraction pectins as side chains. Carbohydrate Polymers, 197(1), 395-402. PMid:30007628. http://dx.doi.org/10.1016/j.carbpol.2018.06.022

Kobori, C. N. (2010). Composição e estabilidade de carotenoides em alimentos (Tese de doutorado). Faculdade de Engenharia de Alimentos, Universidade Estadual de Campinas, Campinas. Recuperado em 16 de dezembro de 2019, de http://repositorio.unicamp.br/bitstream/REPOSIP/256124/1/Kobori_CintiaNanci_D.pdf

Kuskoski, E. M., Asuero, A. G., Morales, M. T., \& Fett, R. (2006). Frutos tropicais silvestres e polpas de frutas congeladas: atividade antioxidante, polifenóis antocianinas. Ciência Rural, 36(4), 1283-1287. http://dx.doi.org/10.1590/S010384782006000400037

Lima, J. F., Silva, M. P. L., Teles, S., Silva, F., \& Martins, G. N. (2010). Avaliação de diferentes substratos na qualidade fisiológica de sementes de melão de caroá [Sicana odorifera (Vell.) Naudim]. Revista Brasileira de Plantas Medicinais, 12(2), 163-167. http://dx.doi.org/10.1590/S1516-05722010000200007

Madeira, N. R. (2016). Sicana odorifera: croá. In R. F. Vieira, J. Camillo \& L. Coradin (Eds.), Espécies nativas da flora brasileira de valor econômico atual ou potencial: Plantas para o futuro: Região Centro-Oeste (1160p.). Brasília: MMA.

Melo, E. A., Maciel, M. I. S., Lima, V. L. A. G., \& Nascimento, R. J. (2008). Capacidade antioxidante de frutas. Brazilian Journal of Pharmaceutical Sciences, 44(2), 201. http://dx.doi.org/10.1590/S1516-93322008000200005

Mineiro, S. A. L. (2014). Fibra alimentar: Composição, métodos e implicações alimentares (Dissertação de mestrado). Faculdade de Ciências e Tecnologia de Nova Lisboa, Portugal.

Morais, D. R., Rotta, E. M., Sargi, S. C., Bonafe, E. G., Suzuki, R. M., Souza, N. E., Matsushita, M., \& Visentainer, J. V. (2017). Proximate composition, mineral contents and fatty acid composition of the different parts and dried peels of tropical fruits cultivated in Brazil. Journal of the Brazilian Chemical Society, 28(2), 308-318. http://dx.doi.org/10.5935/0103-5053.20160178

Moreira, F. (1996). Plantas que curam: Cuide de sua saúde através da natureza (256 p.). São Paulo: Hemus.

Morton, J. F. (1987). Fruits of warm climates (pp. 444-445). Miami: Echo Point Books \& Media. Recuperado em 16 de dezembro de 2019, de https://www.pssurvival.com/ps/plants/Crops_Fruits_Of_Warm_Climates_2004

Nascimento, R. S. M., Cardoso, J. A., \& Cocozza, F. D. M. (2014). Caracterização física e físico-química de frutos de mangabeira (Hancornia speciosa Gomes) no oeste da Bahia. Revista Brasileira de Engenharia Agrícola e Ambiental, 18(8), 856-860. http://dx.doi.org/10.1590/1807-1929/agriambi.v18n08p856-860 
Parada, F., Duque, C., \& Fujimoto, Y. (2000). Free and bound volatile composition and characterization of some glucoconjugates as aroma precursors in Melon de olor fruit pulp (Sicana odorifera). Journal of Agricultural and Food Chemistry, 48(12), 6200-6204. PMid:11312792. http://dx.doi.org/10.1021/jf0007232

Priori, D., Barbieri, R. L., Neitzke, R. S., Vasconcelos, C. S., Oliveira, C. S., Mistura, C. C., \& Costa, F. A. (2006). Banco ativo de germoplasma de germoplasma de cucurbitáceas da Embrapa Cucurbitáceas da Embrapa Clima Temperado - período de 2002 a 2002 a 2006. Embrapa.

Quettier-Deleu, C., Gressier, B., Vasseur, J., Dine, T., Brunet, C., Luyckx, M., Cazin, M., Cazin, J.-C., Bailleul, F., \& Trotin, F. (2000). Phenolic compounds and antioxidant activities of buckwheat (Fagopyrum esculentum Moench) hulls and flour. Journal of Ethnopharmacology, 72(1-2), 35-42. PMid:10967451. http://dx.doi.org/10.1016/S0378-8741(00)00196-3

Reis, P. S., Ferreira, S. L. C., Carvalho, R. D. S., \& Conceição, M. F. B. (2009). Determinação da composição centesimal da polpa do melão coroá (Sicana odorifera Naud.). In Anais da XXXII Reunião Anual da Sociedade Brasileira de Química. Fortaleza.

Ribeiro, D., Freitas, M., Silva, A. M. S., Carvalho, F., \& Fernandes, E. (2018). Antioxidant and pro-oxidant activities of carotenoids and their oxidation products. Food and Chemical Toxicology, 120(6), 681-699. PMid:30077704. http://dx.doi.org/10.1016/j.fct.2018.07.060

Rigolon, T. C. B. (2017). Predição do conteúdo de antocianinas, fenólicos totais e capacidade antioxidante dos frutos de amora (Rubus sp.), Mirtilo (Vaccinium sp.) E casca de jabuticaba (Plinia jaboticaba) usando parâmetros colorimétricos (Dissertação de mestrado). Universidade Federal de Viçosa, Viçosa.

Rochelle, L. A. (1986). Contribuição ao conhecimento do Cruá (Sicana odorifera Naud.). Anais da Escola Superior de Agricultura Luiz de Queiroz, 43(2), 378-388. http://dx.doi.org/10.1590/S0071-12761986000200004

Rodriguez-Amaya, D. B. (2001). A guide to carotenoid analysis in foods. Washington D.C.: International Life Sciences Institute OMNI Press.

Rufino, M. S. R., Alves, R. E., de Brito, E. S., Pérez-Jiménez, J., Saura-Calixto, F., \& Mancini-Filho, J. (2010). Bioactive compounds and antioxidant capacities of 18 non-traditional tropical fruits from Brazil. Food Chemistry, 121(4), 996-1002. http://dx.doi.org/10.1016/j.foodchem.2010.01.037

Silva, J., Silva, E. S., \& Silva, P. S. L. E. (2002). Determinação da qualidade e do teor de sólidos solúveis nas diferentes partes do fruto da pinheira (Annona squamosa L.). Revista Brasileira de Fruticultura, 24(2), 562-564. http://dx.doi.org/10.1590/S010029452002000200057

Singleton, V. L., Orthofer, R., \& Lamuela-Raventós, R. M. (1999). Analysis of total phenols and other oxidation substrates and antioxidants by means of folin-ciocalteu reagent. Methods in Enzymology, 299, 152-178. http://dx.doi.org/10.1016/S00766879(99)99017-1

Souza, C. O., Menezes, J. D. S., Ramos, N. D. C., Assis, J. G. A., Silva, S. R., \& Druzian, J. I. (2012). Carotenoides totais e vitamina A de cucurbitáceas do Banco Ativo de Germoplasma da Embrapa Semiárido. Ciência Rural, 42(5), 926-933. http://dx.doi.org/10.1590/S0103-84782012005000024

Sucupira, N. R., Silva, A. B., Pereira, G. J. N., \& Costa, G. (2012). Métodos para a determinação da atividade antioxidante de frutos. UNOPAR Científica. Ciências Biológicas e da Saúde, 14(4), 263-269. http://dx.doi.org/10.17921/24478938.2012v14n4p\%25p

Swain, T., \& Hillis, W. E. (1959). The phenolic constituents of prunusdomestica. The quantitative analysis of phenolic constituents. Journal of the Science of Food and Agriculture, 10(1), 63-68. http://dx.doi.org/10.1002/jsfa.2740100110

Tamer, C. E., İncedayi, B., Parseker Yönel, S., Yonak, S., \& Çopur, O. U. (2010). Evaluation of several quality criteria of low calorie pumpkin dessert. 56 Notulae Botanicae Hort. Agrobotanici, Cluj-Napoca, 38(1), 76-80.

Torres, D. F. G., Assunção, D., Mancini, P., Torres, R. P., \& Mancini-Filho, J. (2002). Antioxidant activity of macambo (Theobroma bicolar L) extracts. European Journal of Lipid Science and Technology, 104(5), 278-281. http://dx.doi.org/10.1002/1438-9312(200205)104:5<278::AID-EJLT278>3.0.CO;2-K

Universidade Estadual de Campinas - UNICAMP. (2011). TACO: Tabela brasileira de composição de alimentos (4. ed.). Campinas: UNICAMP. Recuperado em 2 de outubro de 2018, de http://www.unicamp.br/nepa/taco/contar/taco_4_edicao_ampliada_e_revisada.pdf?arquivo=ta co_4_versao_ampliada_e_revisada.pdf 